\title{
DOCENTES, TAREAS Y ALUMNOS EN LA DEFINICIÓN DEL COMPROMISO: INVESTIGANDO EL AULA DE NIVEL PRIMARIO DE EDUCACIÓN
}

\author{
Daiana Yamila Rigo* \\ Universidade Nacional de Río Cuarto (UNRC), Río Cuarto, Córdoba, Argentina
}

RESUMEN: El artículo presenta resultados preliminares de una investigación desarrollada con alumnos y docentes de nivel primario en el área de las Ciencias Sociales. El propósito fue describir, explorar y explicar cómo el compromiso de los estudiantes se relaciona e interrelaciona con factores contextuales y la configuración de la clase. Se entiende por implicación la intensidad y la emoción con las cuales los estudiantes se involucran en el contexto de enseñanza para iniciar y llevar a cabo actividades de aprendizaje. Para la recolección de datos, se usaron cuestionarios y protocolos de observación. Los resultados muestran que la configuración de la clase se define como factor clave para atender al compromiso, asimismo, las principales conclusiones permiten apreciar que el compromiso se contagia; y el contexto de aprendizaje y el diseño de la clase son fundamentales en la implicación que los alumnos muestran hacia el aprendizaje en el área de las Ciencias Sociales.

Palabras clave: Educación Primaria. Compromiso. Contexto Instruccional. Soporte Docente.

\section{DOCENTES, TAREFAS E ALUNOS NA DEFINIC̣ÃO DE COMPROMISSO: PESQUISANDO A SALA DE AULA DE ENSINO FUNDAMENTAL}

RESUMO: $\mathrm{O}$ artigo apresenta resultados preliminares de uma pesquisa desenvolvida com alunos e docentes do Ensino Fundamental na área de Ciências Sociais. O propósito foi descrever, explorar e explicar como o compromisso dos estudantes está relacionado a fatores contextuais e à configuração da classe, e como compromisso, contexto e classe se correlacionam. Entende-se por implicação a intensidade e a emoção presentes no envolvimento dos estudantes com o contexto de ensino para iniciarem e levarem adiante atividades de aprendizagem. Para a coleta de dados, usaramse questionários e protocolos de observação. Os resultados mostram que a configuração da classe se define como fator-chave para trazer à tona a noção de compromisso; as principais conclusões permitem apontar que o compromisso pode ser disseminado e que o contexto de aprendizagem e a organização da classe têm um papel fundamental no nível de envolvimento que os alunos demonstram para a aprendizagem na área de Ciências Sociais. Palavras-chave: Ensino Fundamental. Compromisso. Contexto Instrucional. Suporte Docente.

'Dra en Psicología. Profesora en la Universidad Nacional de Río Cuarto. Investigadora Asistente del CONICET. E-mail: < daianarigo@hotmail.com>. 


\section{TEACHERS, TASKS AND STUDENTS IN THE DEFINITION OF ENGAGEMENT: RESEARCHING THE CLASSROOM PRIMARY EDUCATION}

ABSTRACT: The article presents preliminary results of research conducted with students and teachers at primary level in the area of Social Sciences. The purpose was to describe, explore and explain how student engagement relates to the contextual factors and teaching style of classroom and how engagement, context and classroom interact themselves. Understanding by involvement, the intensity and the emotion with which the students are involve for start and carry out learning activities. Data collection have used questionnaires and observation protocols. The results have shown that the classroom configuration is defined as key factor to address and understand the engagement; Also, the main conclusions can point the engagement can be shared; and the learning context and classroom organization play a fundamental role in the level of involvement that students show towards learning in the area of Social Sciences.

Keywords: Primary Education. Engagement. Instructional Context. Teacher Support.

\section{INTRODUCCIÓN}

A menudo nos detenemos a observar solo una parte de la clase, a los alumnos, las tareas académicas o los docentes, pero en menor medida se ha investigado la relación entre estas dimensiones para comprender el compromiso. Por lo general, se está atento al comportamiento de los estudiantes, si participan, formulan preguntas, solicitan aclaraciones o inician debates; al estilo del docente para dictar la clase; o al formato de las actividades y al tiempo dedicado a la realización de trabajos prácticos (HARRIS, 2010; LAM et al. 2012; RIGO; DONOLO, 2014).

Estas particularidades de alumnos, docentes y tareas interactúan entre sí en ambientes reales de aprendizaje configurando el contexto instruccional. Turner y Meyer (2000) mencionan que por muchos años en Psicología de la Educación no solo se evitó el estudio del contexto, sino que también se estudió como constructos separados la enseñanza y el aprendizaje. Asimismo, los investigadores tendieron a aislar las variables más que tratar de comprender la compleja relación entre las dimensiones en cuestión. Sin embargo, actualmente, se vislumbra que, para estudiar el contexto de la clase, se requiere la investigación de más de una variable al mismo tiempo para alcanzar una mayor comprensión y a la vez usar diferentes métodos para su estudio y abordaje. Retomando los postulados de Piaget, quien promovió la idea de la interacción entre el sujeto y el objetivo en la construcción del conocimiento, que 
trasladada al contexto pedagógico, se percibe la importancia de estudiar las interrelaciones entre los alumnos, los docentes y el contexto donde las tareas académicas se llevan a cabo (CASTORINA et al., 1998).

$\mathrm{Al}$ respecto, también Good (2010), en una revisión sobre estudios acerca de la clase, reflexiona que no han sido muchos los avances en los últimos 40 años, sobre todo en la capacidad para mejorar los desarrollos teóricos y empíricos que expliquen cómo los maestros impactan sobre el aprendizaje de los alumnos. A la vez, se interroga sobre qué marca la diferencia en un docente efectivo y responde subrayando la importancia de contemplar distintas dimensiones al mismo tiempo, enfatizando una enseñanza que soporte el aprendizaje de los estudiantes a partir de la facilitación de un currículo pensado para generar oportunidades para aprender y participar de manera activa en la construcción de significados, a fin de aplicarlos a nuevos contextos.

En el marco de estos nuevos desafíos en investigación educativa, específicamente en este trabajo, estamos interesados en conocer cómo las características de los alumnos, los docentes y las tareas interrelacionadas ayudan a comprender el compromiso como un metaconstructo que demanda estudios contextualizados en las aulas y desde los primeros años de escolarización para que, a partir de los resultados derivados de estudios empíricos, se pueda planificar acciones que lo promuevan. Su importancia radica sobre todo si pensamos en las principales problemáticas que se presentan como más relevantes en los distintos niveles educativos: falta de interés, aburrimiento, desmotivación, entre otros de igual tenor.

\section{¿QUÉ ES EL COMPROMISO? CONCEPTUALIZACIONES CENTRALES}

Las investigaciones iniciales entienden que el compromiso, también denominado implicación o engagement, es la inversión psicológica en y hacia el aprendizaje, la comprensión, o dominio de conocimiento, las habilidades o destrezas que el trabajo académico intente promover (NEWMANN; WEHLAGE; LAMBORN, 1992). Podría decirse que es el grado en que los alumnos están implicados, conectados y comprometidos activamente para aprender y rendir, en contraste con la participación superficial, la apatía y la falta de interés. Más específicamente el compromiso hacia las tareas académicas se refiere a la intensidad y emoción con las cuales los estudiantes se implican para iniciar y llevar a cabo actividades de aprendizaje; es una energía en acción que conecta a la persona con la actividad (APPLETON et al., 2006; MITCHELL; CARBONE, 2011; RIGO, 2014; RIGO; DONOLO, 2014). 
Compromiso es un concepto multidimensional, un metaconstructo, integrado por tres componentes, a saber: cognitivo, conductual y afectivo. Y está relacionado respectivamente con la manera cómo los estudiantes se comportan y se sienten acerca de la experiencia educativa, el tipo de tareas académicas, las posibilidades de participar, elegir y profundizar y el interés que tienen sobre los trabajos realizados en la clase (ARGUEDAS, 2010; FREDRICKS; BLUMENFELD; PARIS, 2004).

El compromiso cognitivo refleja el grado en el cual los alumnos están atendiendo y dedicando esfuerzos hacia el aprendizaje, la comprensión, el dominio disciplinar y la adquisición de habilidades que el trabajo académico intenta promover. Un alumno comprometido en este nivel es un estudiante estratégico y que regula autónomamente los procesos de aprendizaje. Con ello se quiere decir que a la hora de aprender se puede adoptar, o bien un enfoque superficial, o un enfoque profundo que permita integrar el nuevo contenido con los conocimientos previos y hacer uso o no de estrategias metacognitivas para planificar, monitorear y evaluar las tareas y las metas de logro (RIGO, 2013, 2014).

$\mathrm{El}$ compromiso conductual ha sido definido contemplando, al menos, tres dimensiones. La primera se refiere a las conductas positivas, tales como seguir reglas, respetar las normas de la clase, así como, ausencia de conductas disruptivas. La segunda está relacionada con la implicación en el aprendizaje y las tareas académicas, lo cual incluye participación en clase, persistencia, concentración, atención, responder preguntas, hacer preguntas y contribuir en las discusiones de la clase. Y la tercera involucra la participación en actividades extraescolares, tales como practicar algún deporte, participar del centro de estudiantes del colegio o tomar clases de arte (ARGUEDAS, 2010; FREDRICKS; BLUMENFELD; PARIS, 2004; KONG; WONG; LAM, 2003; MITCHELL; CARBONE, 2011).

Otra consideración sobre el componente conductual de la implicación es realizada por Finn (1993), quien entiende que la participación es el concepto clave para el estudio de este constructo e identifica cuatro niveles. El primero incluiría conductas básicas, tales como la asistencia regular, atender a las normas y directrices de docentes. El segundo abarcaría las conductas anteriores más las iniciativas del alumno, como plantear preguntas, iniciar una discusión y buscar ayuda. El tercero comprendería la participación del alumno en aspectos sociales o actividades extracurriculares. Y por último, un cuarto nivel referido a la participación de los estudiantes en la organización escolar indicando mayor implicación e identificación con la escuela (GONZÁLEZ GONZÁLEZ, 2010). 
El compromiso afectivo está integrado por las reacciones negativas y positivas de los alumnos hacia los profesores, los compañeros de clase y las tareas académicas. En general, un buen indicador de compromiso emocional es el interés hacia el trabajo en el aula; a la inversa, menor implicación afectiva sería afín a conductas tales como el aburrimiento, la ansiedad, la frustración y los nervios en el aula. Al respecto, Eccles et al. (1983) describe cuatro componentes, que son: el interés asociado al disfrute de las actividades, el valor relacionado al logro de concluir la tarea, el valor instrumental de la tarea vinculado a la importancia para conseguir metas futuras y el costo, es decir, los aspectos negativos de participar en la tarea (KONG; WONG; LAM, 2003).

\section{Alumnos comprometidos, docentes implicados y tareas que involucran}

Como formulábamos en la introducción, las nuevas líneas de investigación buscan responder al desafío de estudiar más de una variable al mismo tiempo. En este sentido, los modelos teóricos y empíricos buscan explicaciones que van más allá de las características individuales; específicamente tratan de comprender cómo interaccionan los factores personales e instructivos para describir y explicar el compromiso de los alumnos hacia el aprendizaje. Al respecto, Shernoff (2013) entiende que si bien el compromiso es un constructo multidimensional influenciado a nivel micro por las relaciones con pares-familia-escuela y a nivel macro por los factores socioeconómicos, sólo una cuarta parte de su variación se explica por factores internos al estudiante, el resto se atribuye a factores del entorno.

A nivel general, en el campo de la Psicología de la Educación, estos planteamientos están en la línea de las investigaciones de Bandura (1986) y Schunk y Pajares (2004), quienes desde un enfoque sociocognitivo comprenden que el aprendizaje involucra la interacción entre cogniciones individuales y características del entorno educativo. A nivel más específico, en los estudios sobre implicación, el modelo contextual de Lam et al. (2012) explica cómo se definen las interrelaciones entre rasgos de los alumnos, docentes y tareas. Los autores se refieren a las influencias recíprocas entre los rasgos que definen al contexto instruccional, las relaciones sociales, los factores personales y los resultados de aprendizaje. Entre los primeros se destaca la importancia de seis componentes que han sido reportados por estudiantes comprometidos; se ha identificado que las tareas ofrecidas por el docente que sean desafiantes, relacionadas con la vida cotidiana y que promuevan la curiosidad y la autonomía, así como el reconocimiento y la evaluación justa, son indicadas como factores 
asociados a altos niveles de implicación. Por su parte, refiriéndose a las relaciones interpersonales, destacan que los estudiantes con mayor compromiso perciben un alto grado de soporte por parte de docentes, pares y familia, por el contrario una correlación inversa se encuentra con altos niveles de agresión en las relaciones entre pares.

Con respecto a los factores personales, el estudio de Lam et al. (2012) muestra que las creencias motivacionales, tales como autoeficacia, metas de aprendizaje orientadas al dominio y atribuciones dirigidas al esfuerzo, se muestran más afín al perfil que exhiben los alumnos que estarían más involucrados en la tarea de aprender. Y con relación al rendimiento académico, encuentra que los estudiantes más implicados a nivel cognitivo, afectivo y conductual son los que tienden a presentar resultados positivos en sus tareas.

Por su parte, la revisión reportada por Shernoff (2013) es coincidente en algunos puntos. En general, los estudios sobre factores externos muestran que los alumnos con alto nivel de compromiso tienden a percibir al contexto de la clase como desafiante, disfrutable, importante, con alto grado de autonomía y nivel de actividad. Estos resultados muestran la importancia de combinar intensidad académica y respuesta emocional positiva en la planificación de las tareas académicas, es decir, no es suficiente sólo disfrutar (play-like engagement) del trabajo del aula, sino también, sobre todo, percibirlo como desafiante (work-like engagement) para promover un Compromiso significativo con la C mayúscula.

Otro rasgo encontrado que relaciona alto compromiso con características contextuales es el valor de utilidad otorgado a la tarea. $\mathrm{Al}$ respecto, los resultados encontrados parecen convenir en que los alumnos que perciben que las actividades académicas son útiles y relevantes más allá de la situación inmediata de realización, son aquellos que tienden a adoptar metas orientadas al dominio, desarrollar mayor interés y compromiso hacia el aprendizaje (BLUMENFELD; KEMPLER; KRAJCIK, 2006; ECCLES et al., 1983; ECCLES; WIGFIELD, 2002; STIPEK, 1996).

Dos características más han sido descriptas por los alumnos comprometidos sobre factores externos asociados al proceso de enseñanza-aprendizaje. Por un lado, la autonomía, que se refiere a la oportunidad de elegir y tener un rol significativo en la dirección de la tarea, es decir, tomar decisiones sobre el tópico a estudiar, seleccionar y planificar la actividad y elaborar el producto final. Al respecto, Ames (1992), Blumenfeld, Kempler y Krajcik (2006) y Stipek (1996) entienden que estas prácticas son afines a respuestas positivas orientadas a incrementar el interés de los estudiantes por el estudio. 
Por otro lado, la curiosidad, articulada a la posibilidad de explorar e indagar más allá de la consigna, supone ofrecer instancias pedagógicas específicas para incentivar al estudiante a formular preguntas o pensar sobre fenómenos que sean intrigantes (LAM; PAK; MA, 2007).

En lo que respecta a la evaluación de los aprendizajes y el reconocimiento del esfuerzo realizado por aprender rescatados en el modelo de Lam et al. (2012), los estudios reportan que la forma en que son evaluados los alumnos afecta el compromiso de estos. Por su parte, Ames (1992) distingue entre la evaluación orientada al logro y la evaluación orientada al aprendizaje, a tal efecto que los alumnos más implicados valoran más las apreciaciones orientadas al dominio que las dirigidas a la norma. En consonancia, Stipek (1996) y Nichols y Dawson (2012) acuerdan que las evaluaciones formativas, y no tanto las sumativas, se conectan con procesos relacionados con la mejora del compromiso.

En relación con el estilo docente y las características que promueven el compromiso en los alumnos, encontramos que aquellos maestros que ofrecen instancias de feedback no solo facilitan el aprendizaje de los estudiantes, sino también el compromiso cognitivo y conductual de los educandos, en tanto se considera desde una perspectiva vigotskiana que el aprendizaje es un proceso transaccional entre la persona y el medio ambiente (PIANTA; HAMRE; ALLEN, 2012; SHERNOFF, 2013). Las investigaciones también sugieren que aquellos docentes que configuran la clase proveyendo instancias para que sus alumnos participen, interactúen e intercambien ideas con los demás durante las tareas académicas, facilitan el desarrollo de la capacidad para justificar la propia perspectiva y atender a las opiniones de los demás, un aspecto que Ryan y Patrick (2001) entienden que promociona el compromiso, la eficacia académica y el aprendizaje autorregulado de los estudiantes.

Además, aparece como concepto clave asociado a la clase y a mayor compromiso el soporte que ofrece el docente a los estudiantes y sus necesidades, rescatándose principalmente la noción de andamiaje, la cual implica lograr un adecuado equilibrio entre las posibilidades de autonomía y el ofrecimiento de una estructura que ayude al estudiante a progresar y llegar a un alto nivel de comprensión (REEVE et al. 2004; RYAN; PATRICK, 2001; SCHUSSLER, 2009; SHERNOFF, 2013). Asimismo, Hipkins (2012) considera que no basta con que los docentes establezcan oportunidades para aprender — tal cual es orquestada por el educador- a fin de generar compromiso en los estudiantes, él plantea que la selección de determinados contenidos y recursos educativos tiene que estar ligada a la percepción de los estudiantes sobre las 
posibilidades de acción —affordances- que ofrece ese entorno para aprender, motivarse e interactuar con sus conocimientos y experiencias previas. Por esto pareciera importante considerar en la planificación de la clase los perfiles intelectuales de los estudiantes, sus fortalezas y competencias. Sobre esto último, Smith (2009 citado por SHERNOFF, 2013) postula como un componente fundamental de la clase para mantener el compromiso emocional de los estudiantes contemplar las diferentes inteligencias múltiples ofreciendo a los sujetos recursos didácticos variados con los cuales interactuar y optar para aprender.

Pareciera de igual importancia la configuración del contexto instructivo orientado al alumno y no tanto al contenido y hacia metas de logro que promueven el dominio más que el desempeño (HUGHES; WU; WEST, 2011; OPDENAKKER; DAMME, 2006). Al respecto, Schussler (2009) indica que tener como pilares en la educación tanto la monotonía como el interés por querer finalizar la tarea promueve menos compromiso y participación de los estudiantes. Por el contrario, cuando los estudiantes perciben que tienen oportunidades para tener éxito en tareas auténticas a través de una instrucción flexible, existen más chances de propiciar la participación; asimismo, los profesores que estructuran el aula para facilitar la participación de los estudiantes, son aquellos que demuestran entusiasmo por el contenido y hacen uso de estrategias de enseñanza a propósito para captar el interés de los estudiantes.

La investigación de Harris (2010) muestra que los maestros tienen distintas representaciones sobre cómo propiciar entornos de aprendizajes dirigidos a aumentar la implicación a sus alumnos. En sus resultados considera tres categorías: entregar, modificar y colaborar. En la primera, los docentes consideran que el modelo de trasmisión centrado en el docente es aquel que posibilita la promoción del compromiso en los educandos, consideran a los alumnos como pasivos, receptores de la información y el contenido, e imparten una instrucción homogénea sin diversificar ni considerar el interés del grupo aula prescribiendo actividades. En la segunda, se encuentra un grupo de docentes que entienden que la clave está en modificar el currículo en función de los intereses de los estudiantes y de esa forma promover la participación de éstos, adaptando las actividades y encontrando materiales adecuados al grupo de la clase, y así llegar a cada estudiante. Por último, en la tercera, los docentes piensan que, para facilitar la implicación, deben programar la clase de manera colaborativa junto a los estudiantes en función de los propósitos y metas de los alumnos, dado que contribuye a la apropiación del conocimiento, al desarrollo de un pensamiento crítico y a un aprendizaje profundo, donde el educando es comprendido como sujeto activo que toma decisiones 
en su proceso de aprendizaje. Sobre esta última categoría, la autora entiende que es la forma más compleja para facilitar el compromiso de los estudiantes, sin embargo enfatiza en la necesidad de seguir profundizando en el estudio y los resultados encontrados.

Desde este marco conceptual, la presente investigación se indaga y busca respuestas sobre las características de la clase y el compromiso de los alumnos. Presentamos resultados preliminares para empezar a entender las diferentes dimensiones que contribuyen a implicarse en el proceso de enseñanza-aprendizaje.

\section{METODOLOGÍA}

Con el objetivo de describir y explicar cómo el diseño de la clase contribuye al desarrollo del compromiso de los estudiantes en el área de las Ciencias Sociales, llevamos a cabo una investigación que indaga sobre cinco dimensiones que se ponen en juego en el aula cuando se enseña y se aprende y que definen la implicación en el contexto escolar formal: el docente y la clase, el compromiso cognitivo, conductual y afectivo, y el contexto instructivo.

\section{¿Con quiénes y cómo lo bicimos?}

La muestra del estudio estuvo conformada por cuatro docentes, uno por clase, y 83 alumnos de sexto grado de Educación Primaria, Localidad Las Higueras, Córdoba, Argentina, distribuidos de la siguiente manera:

\begin{tabular}{|l|l|}
\hline \multicolumn{1}{|c|}{ Clase } & \multicolumn{1}{c|}{ Número de alumnos } \\
\hline 1 & 14 \\
\hline 2 & 19 \\
\hline 3 & 26 \\
\hline 4 & 24 \\
\hline
\end{tabular}

La recolección de datos se llevó a cabo en el área de Ciencias Sociales durante el primer semestre de los años 2012, 2013 y 2014. Cada instrumento se administró en horario de clase junto a la presencia del docente tutor del aula y con previa autorización de directivos y padres bajo el consentimiento informado de los alumnos. Se informó a las respectivas autoridades y estudiantes el objetivo de la investigación y el tiempo destinado al rellenado de los protocolos - no mayor a 60 minutos-. Las observaciones no participantes se realizaron tres veces por semana abarcando la carga horaria total de la materia en estudio; ellas fueron realizadas por un profesional 
experto haciendo uso de una planilla confeccionada a tal finalidad. Asimismo, se siguieron las pautas de consentimiento informado y, para resguardar la confiabilidad de los datos y el anonimato a cada sujeto, se le asignó un código.

¿Cómo se registraron, recolectaron y analizaron los datos?

\section{Configuración de la clase}

Para su valoración durante el ciclo lectivo 2012, 2013 y 2014, en el primer semestre, se llevaron a cabo observaciones no participantes con la finalidad de obtener información sobre la clase, tal cual era configurada y planificada por el docente. En total se observaron cuatro aulas con diferentes docentes que impartían la asignatura Ciencias Sociales en sexto grado de nivel primario de educación. Los indicadores que se tuvieron en cuenta para llevar a cabo los registros fueron las siguientes seis dimensiones, elaboradas a partir de la bibliografía consultada y desarrollada en el marco teórico del presente artículo:

1. Retroalimentación. Se observó si los docentes durante la clase ofrecían feedback a sus alumnos. No interesaba tanto la cantidad de interacción entre docente y alumnos, sino la calidad de éste. Se atendió a los intercambios sobre información específica no solo sobre si la tarea era correcta, sino también sobre cómo podrían mejorar el trabajo para enriquecer sus producciones, alcanzar sus metas o repensar la actividad en pro de conseguir una mejor comprensión y aprendizaje, favoreciendo la autoevaluación y la regulación de la cognición. Asimismo, atendiéndose a que las retroalimentaciones resalten el esfuerzo y el progreso personal del estudiante, a modo de promover atribuciones causales internas y controlables, más que externas e incontrolables.

2. Soporte docente. Se registró durante las observaciones si el docente proveía tanto soporte de autonomía, es decir, si ofrecía al estudiante la posibilidad de elegir, seleccionar y controlar aspectos en relación con la toma de decisiones en la tarea y su resolución, tales como la elección del tema, la forma de trabajarlo y la manera de presentarlo; como si brindaba soporte instrumental o estructura a los estudiantes, o sea, si el docente ofrecía a los estudiantes instrucciones claras, comprensibles, explícitas y detalladas, si brindaba un programa 
de acción para guiar la actividad de los alumnos y si orientaba al estudiante a través de preguntas destinadas a ayudar a que progresara y alcanzara un mayor nivel de comprensión.

3. Oportunidad para aprender. Se observó si las clases planificadas por el docente mostraban rasgos que dieran cuenta de contemplar los intereses de los estudiantes, fundamentalmente se registró que lainstrucción considerara y pusiera en juego una multiplicidad de recursos didácticos a fin de abarcar las diferencias individuales de los sujetos en términos de perfiles intelectuales, se tuvieran en cuenta las propuestas de los estudiantes y sus inquietudes.

4. Participación. Se consideró en las observaciones si el maestro ofrecía instancias para que los estudiantes tuvieran lugar de expresar su opinión, iniciar discusiones y debates, aportar información y compartir en el grupo de pares, consultar y expresar dudas, participar en clase y en las tareas activamente, y tener la posibilidad de argumentar y justificar sus ideas.

5. Estructura de la clase. Se registró que las intervenciones del docente estuvieran orientadas a promover una enseñanza centrada en el alumno, considerando a este como sujeto activo, asimismo, que el profesor con su participación ofreciera motivos y oportunidades para desarrollar metas orientadas al dominio más que al desempeño.

6. Evaluación. Se observó si el docente explicitaba los criterios y objetivos de valoración, asimismo, si las diversas instancias estaban orientadas a guiar el proceso, proporcionando información significativa acerca del aprendizaje de los estudiantes y apoyando sus decisiones, más que atender sólo al producto final.

Los datos fueron reunidos en una planilla confeccionada para tal finalidad y el investigador iba marcando aquellas características que se cumplían o no en la clase de cada docente. La presencia de los indicadores en una clase se relaciona con un diseño orientado a promover el compromiso, mientras que la ausencia de estos sugiere que la clase planificada por el maestro no se enmarca dentro de las características teóricas que facilitan la implicación de los alumnos hacia el aprendizaje y las tareas desarrolladas en el área de las Ciencias Sociales. Se consideró la siguiente escala para computar la frecuencia con que cada dimensión estaba presente en la clase: (A) Siempre: entre el 90\% y el 100\%; (B) Generalmente: entre el 50\% y el $90 \%$. (C) Casi nunca: entre el 20\% y el 40\%. (D) Nunca: menos del 10\%. 


\section{Compromiso cognitivo}

El compromiso cognitivo fue evaluado a partir de 13 ítems que indagaban sobre el uso de estrategias profundas - EP- (7 ítems) y estrategias superficiales -ES - (6 ítems) en la hora de Ciencias Sociales (ejemplos: "En el aprendizaje de las Ciencias Sociales, siempre intento plantearme preguntas porque estas me ayudan a comprender aspectos centrales de las Ciencias Sociales" versus "Encuentro que memorizar información es la mejor forma de aprender Ciencias Sociales"). Estos fueron adaptados de la escala The Student Engagement in the Mathematics Classroom Scale de Kong, Wong y Lam (2003) al castellano y al área disciplinar de interés. Los estudiantes estimaron su implicación escogiendo una alternativa de respuesta considerando que 1 indicaba un mayor uso de esa estrategia, mientras que 5 implicaba un empleo mucho menor.

Para establecer el nivel de compromiso cognitivo de los alumnos se tomó como punto de corte la mediana $\left(\mathrm{EP} \mathrm{M}_{\mathrm{e}}=23\right.$; $\mathrm{ES}$ $\mathrm{M}_{\mathrm{e}}=17$ ), permitiendo conformar cuatro grupos, por un lado, los que percibían hacer más uso de estrategias superficiales o profundas; por otro lado, los que tenían una percepción de utilizar con menor frecuencia estrategias de tipo profundas o superficiales.

\section{Compromiso conductual}

Para evaluarlo, se procedió durante un semestre de los años 2012, 2013 y 2014 a realizar observaciones no participantes de tres cursos durante la clase de Ciencias Sociales, con el objetivo de recopilar información sobre indicadores que dieran cuenta del compromiso conductual de cada alumno. En total se consideraron los nueve criterios siguientes, a saber: responder a las preguntas del profesor; preguntar dudas o solicitar aclaraciones al profesor; escuchar y atender a la exposición del profesor; participar en la clase y en las tareas activamente; contribuir en las discusiones; finalizar y cumplir con las tareas propuestas; permanecer y persistir en la tarea; buscar información extra; colaborar y compartir información traída del hogar. Para su formulación, se contempló las sugerencias ofrecidas por Alexander (2006), Ruhe (2007) y Kong, Wong y Lam (2003), Finn y Zimmer (2012). Cabe destacar que se optó por esta modalidad de evaluación, en tanto la bibliografía específica sobre enfoques e instrumentos de medición indica que la observación del comportamiento orientado hacia la tarea es pertinente para estimar esta dimensión de compromiso (FINN; ZIMMER, 2012; FREDRICKS et al., 2004; FREDRICKS; MCCOLSKEY, 2012; SHERNOFF, 2013). 
Para establecer los niveles de compromiso - muy, algo o poco- se especificó con antelación que la presencia de siete a nueve indicadores sería adecuada para alumnos muy comprometidos, en tanto la presencia de cinco o seis ítems se asociaría a un nivel intermedio y la observación de cuatro o menos criterios en el comportamiento de los alumnos orientados hacia el trabajo académico daría pautas de estar poco comprometido con la tarea en curso.

\section{Compromiso afectivo}

El compromiso afectivo de los alumnos fue evaluado por seis ítems que consultaban sobre el interés y el gusto por aprender (ejemplo: "En la clase de Ciencias Sociales, creo que los contenidos que aprendo son interesantes y agradables"), adaptados de la escala The Student Engagement in the Mathematics Classroom Scale de Kong, Wong y Lam (2003) al castellano y al área disciplinar de interés. Los estudiantes estimaron su implicación en Ciencias Sociales en una escala con valores que oscilaban entre 1 (siempre) y 5 (casi nunca). Para establecer los niveles de compromiso afectivo de los alumnos, se tomó como punto de corte la mediana $\left(\mathrm{M}_{\mathrm{e}}=15\right)$, permitiendo conformar dos grupos, por un lado, los que se percibían muy interesados y, por otro lado, los que tenían una percepción menor sobre su interés en la clase de Ciencias Sociales.

\section{Contexto instructivo}

Para su evaluación, los alumnos respondieron a cinco frases para estimar su apreciación del contexto instructivo. Cada frase consultaba sobre la percepción del entorno educativo refiriéndose a los siguientes aspectos sobre las tareas académicas y las evaluaciones: 1Desafío (Las actividades realizadas eran difíciles, fáciles o desafiantes respecto a tus habilidades y conocimiento); 2- Valor de utilidad (Las tareas y las explicaciones del docente establecían con claridad o no la relación entre el tópico y la vida cotidiana); 3-Curiosidad (Durante la resolución de las tareas el docente ofrecía las respuestas correctas o daba la libertad de explorar y encontrar respuestas alternativas); 4- Autonomía (En las tareas se ofrecía la oportunidad de tomar decisiones y hacer algo distinto o tenías que delimitarte en lo que la consigna solicitaba); 5- Evaluación (Las evaluaciones fueron solo una instancia para poner una nota o el docente ofreció sugerencias para mejorar el trabajo). Las frases fueron redactadas siguiendo los lineamientos y recomendaciones de Lam et al. $(2007,2012)$. En cada caso el alumno debería marcar una de las opciones resultadas en 
negrita para completar la frase e indicar, de ese modo, cómo percibía el contexto instructivo de la clase en Ciencias Sociales.

Respecto a los análisis estadísticos realizados, inicialmente, se llevó a cabo un análisis descriptivo de las variables en estudio, primero se computó la frecuencia con la que cada dimensión de la clase se presentaba en cada aula observada. Luego, para definir la asociación entre las variables, se procedió a aplicar la Prueba Chi-Cuadrado de Pearson y, así, averiguar si existían relaciones estadísticamente significativas entre cada clase y cada una de las variables consideradas para los alumnos. Por último, para explorar y describir la interrelación entre todas las variables, se procedió a realizar un análisis de componentes principales para variables categóricas haciendo uso del modelo de escalamiento óptimo.

\section{RESULTADOS}

A continuación se presentan tres apartados. En el primero se muestran los resultados hallados a partir de las observaciones no participantes realizadas para valorar la clase. En el segundo se presentan, para cada tipo de compromiso y la percepción que del contexto instructivo tiene cada grupo de alumno por clase, las relaciones estadísticamente significativas que, entre las variables, se han encontrado. En el tercero, finalmente, se muestran los resultados derivados del estudio conjunto de las variables para explicar cómo, de manera conjunta e interrelacionada, aclaran el compromiso.

Primera parte - Configuración de la clase

De las observaciones no participantes se obtuvieron los siguientes datos - ver Tabla 1-. Como se puede observar, cada clase presenta características particulares en torno a las seis dimensiones consideradas para valorar cómo el estilo docente y su instrucción promueven el compromiso en los estudiantes. Se espera que la clase que atiende de manera especial a cada una de las dimensiones favorezca un mayor desarrollo de implicación en sus estudiantes hacia el aprendizaje y las tareas académicas.

Entre las cuatro aulas observadas, en la primera encontramos que la maestra ofrecía con mayor frecuencia retroalimentaciones sobre la tarea y sus progresos, un buen andamiaje apoyando el aprendizaje de los estudiantes, ofreciendo soportes para avanzar hacia una mejor comprensión de la actividad, pero brindando, a la vez, espacios para promover autonomía en la toma de decisiones. 
Asimismo, la docente en la clase 1 favoreció el intercambio entre los alumnos creando espacios para iniciar debates, postular ideas, formular dudas y aclaraciones, a la par de configurar un contexto instructivo para orientar a los estudiantes hacia el logro de metas personales tendientes al dominio más que hacia al desempeño y considerando el aprendizaje como individual y activo en función de las competencias de los educandos.

TABLA 1 - Frecuencia porcentual registrada en cada clase para cada dimensión que promueve el compromiso en los estudiantes

\begin{tabular}{|l|c|c|c|c|}
\hline & Clase 1 & Clase 2 & Clase 3 & Clase 4 \\
\hline Retroalimentación & A & C & B & B \\
\hline Soporte docente & A & D & A & C \\
\hline Oportunidad para aprender & B & C & B & B \\
\hline Participación & A & C & A & B \\
\hline Estructura de la clase & A & D & B & B \\
\hline Evaluación & A & D & A & C \\
\hline
\end{tabular}

"Referencia: (A) Siempre: entre el $90 \%$ y el 100\%; (B) Generalmente: entre el $50 \%$ y el $90 \%$; (C) Casi nunca: entre el $20 \%$ y el $40 \%$; (D) Nunca: menos del $10 \%$.

Respecto a la clase 3 , se puede observar que la maestra también estructura oportunidades en el aula que contemplarán las seis dimensiones que desde la teoría posibilitarían mayor implicación a los estudiantes, aunque con menor frecuencia que la clase 1. Y como se muestra en la Tabla 1, en la clase 4 se muestra una configuración que, si bien en muchos aspectos, tiende a promover la implicación, se observa que la dimensión soporte docente no es asistida frecuentemente, además, no se observó que las evaluaciones promovieran mejoras, sino que se orientaban al producto final; y en la clase 2 se tuvo un registro menor en cada dimensión, es decir, la docente no ofrecía con mucha frecuencia un contexto de aprendizaje que tendiera a promover el compromiso de los alumnos en la materia Ciencias Sociales, observándose menos instancias para efectuar intercambios entre alumno y docente, promover la participación de los estudiantes para formular dudas, solicitar aclaraciones, ofrecer argumentos y justificaciones, así como para el desarrollo de competencias personales. De igual manera, se registraron pocas intervenciones de la docente para proveer soportes tanto de autonomía como instrumental que ayudarían a avanzar en la realización de la tarea, así como para promover metas orientadas al dominio. 
Segunda parte - Relación entre tipo de compromiso, percepción del contexto instructivo con diseño de la clase.

A cada estudiante le consultamos sobre su interés y participación en la clase de Ciencias Sociales, asimismo, sobre su percepción del contexto instructivo tal cual fuese configurado por el docente de grado y por el uso de estrategias, o bien superficiales, o profundas para el aprendizaje de la materia en cuestión. A continuación presentamos una descripción de cada variable considerando por separado cada clase y sus respectivos estudiantes. Nos interesa describir cada dimensión de compromiso y la percepción de los alumnos sobre la instrucción por clase observada y a la vez conocer si hay relaciones estadísticamente significativas entre el compromiso reportado por los alumnos en sus tres dimensiones: cognitivo, conductual y afectivo, así como la percepción que estos tienen sobre el contexto instructivo y la clase de pertenencia.

Con respecto a la variable compromiso conductual, entre los estudiantes de la clase 1, encontramos que la mayoría (57\%) se encuentra dentro de la categoría "muy implicados", y un 53\% de los estudiantes de la clase 3 comparte este perfil, mientras que en las clases $2(79 \%)$ y $4(38 \%)$ predominan los estudiantes que están poco comprometidos en el aula y hacia el aprendizaje de las Ciencias Sociales $\left(\mathrm{X}^{2}=28.568, \mathrm{p}=0.001\right)$.

En lo que se refiere a compromiso afectivo, los análisis indican que tanto los alumnos de la clase $1(57 \%)$ como los de la clase $3(61 \%)$ se perciben muy interesados hacia las lecciones ofrecidas por sus maestros, como así también hacia la propuesta didáctica del área de las Ciencias Sociales. Por el contrario, la mayoría de los estudiantes que asisten a la clase $2(79 \%)$ se muestran menos implicados afectivamente, al igual que la mitad de los alumnos de la clase $3(54 \%),\left(X^{2}=7.933, p=0.047\right)$.

En relación a la dimensión cognitiva del constructo compromiso, encontramos que un $79 \%$ de los estudiantes de la clase 1 manifiesta hacer uso de estrategias profundas a la hora de resolver tareas y estudiar contenidos del área de las Ciencias Sociales, de igual manera lo reportan los alumnos de las clases $3(65 \%)$ y $4(75 \%)$, $\left(\mathrm{X}^{2}=27,265, \mathrm{p}=0.001\right)$, en tanto, en la clase $2(84 \%)$, se observa que los educandos tienden a poner en marcha estrategias de aprendizaje de tipo superficiales $\left(X^{2}=20,533, p=0.001\right)$.

Sobre la percepción del contexto instructivo, también hallamos que los estudiantes de las distintas clases tienden a construir una idea diferente sobre las cinco dimensiones consultadas. En la clase 1 la mayoría 
de las respuestas (64\%) acuerdan que las tareas que ofrece el maestro como puente para aprender son desafiantes, es decir, promueven el interés, pero al mismo tiempo se definen dentro de la zona de desarrollo próximo. Entre los alumnos que encuentran que las actividades son fáciles, los alumnos de las clases $2(74 \%)$ y $3(66 \%)$ parecen coincidir en esta percepción, mientras que casi la mitad de los estudiantes $(42 \%)$ de la clase 4 señala que las tareas son difíciles de resolver con relación a sus competencias actuales $\left(\mathrm{X}^{2}=31,410, \mathrm{p}=0.001\right)$.

Asimismo, más del 70\% de los estudiantes de las clases 1 , 3 y 4 aprecian que el valor de utilidad de las tareas realizadas en la materia Ciencias Sociales está presente como característica que promueve la implicación, lo contrario se observa en la clase 2 , donde se registraron menos indicadores relacionados con el desarrollo del compromiso cognitivo, conductual y afectivo $\left(\mathrm{X}^{2}=15,963, \mathrm{p}=0.001\right)$. Con relación a la dimensión curiosidad, los estudiantes que forman parte de las clases $1(79 \%)$ y $3(58 \%)$ perciben que los maestros ofrecen posibilidades de explorar otras respuestas y no tanto buscar una única y correcta solución, tal cual lo aprecian la mayoría de los estudiantes de la clase $2(74 \%)$ y un poco más de la mitad de la 4 $(58 \%),\left(X^{2}=10,106, p=0.018\right)$.

En lo que concierne a la dimensión autonomía, encontramos que la mayoría de los estudiantes de la clase $1(78 \%)$ aprecian tener oportunidades para tomar decisiones, al igual que los alumnos de la clase $3(74 \%)$. Por el contrario, el 58\% de los educandos que integran las clases 2 y 4 perciben menos chances de elección y señalan realizar sólo aquello que la consigna solicita $\left(\mathrm{X}^{2}=8,019, \mathrm{p}=0.046\right)$. Por último, con relación a la evaluación, nuevamente las clases 1 (71\%) y 3 (78\%) parecen ser las que aportan sugerencias para mejorar los productos alcanzados por los estudiantes, mientras que en las clases $2(54 \%)$ y $4(58 \%)$ los alumnos perciben que la nota es el aspecto más valorado por el docente $\left(\mathrm{X}^{2}=9,236, \mathrm{p}=0.026\right)$.

Tercera parte - Interrelaciones entre compromiso, percepción del contexto educativo y configuración de la clase.

Para definir un perfil del análisis conjunto de todas las dimensiones consideradas en el presente estudio, se procedió, a partir del análisis de componentes principales categóricos, a describir las relaciones entre las variables compromiso, contexto instructivo percibido y configuración de la clase. Los resultados indican que el modelo explica el 67\% de la varianza, un 49\% a partir de la Dimensión 1 y tan solo un $18 \%$ a partir de la Dimensión 2. Centrándonos en la primera 
dimensión, la más relevante para su análisis, como se puede observar en la Figura 1, se registran dos agrupaciones centrales. Sobre el eje derecho vertical se indican interrelaciones entre las variables que teóricamente se definen para comprender la promoción del compromiso; encontramos que las dimensiones que definen un contexto educativo con rasgos que implican se agrupan junto a las clases 1 y 3 , donde se encuentran los estudiantes que tienden a estar interesados a participar y hacer uso de estrategias profundas como recursos de aprendizajes centrales. Mientras que, en el eje izquierdo vertical, se muestran agrupadas las variables del contexto instructivo percibido que menos están relacionadas con la facilitación de la implicación cognitiva, conductual y afectiva y que a la vez se corresponden con las características que definen la planificación docente de la clase 2. Cabe destacar que la clase 4 queda explicada por la segunda dimensión, junto a percepciones del contexto instructivo que, por un lado, resulta interesante para los estudiantes y desafiante, pero que, por otro lado, se percibe como muy estructurado y difícil, afectando la participación del alumno en el proceso de aprendizaje.

FIGURA 1 - Diagrama conjunto de puntos para las categorías de configuración de la clase, compromiso cognitivo, conductual y afectivo y variables del contexto instructivo ${ }^{1}$

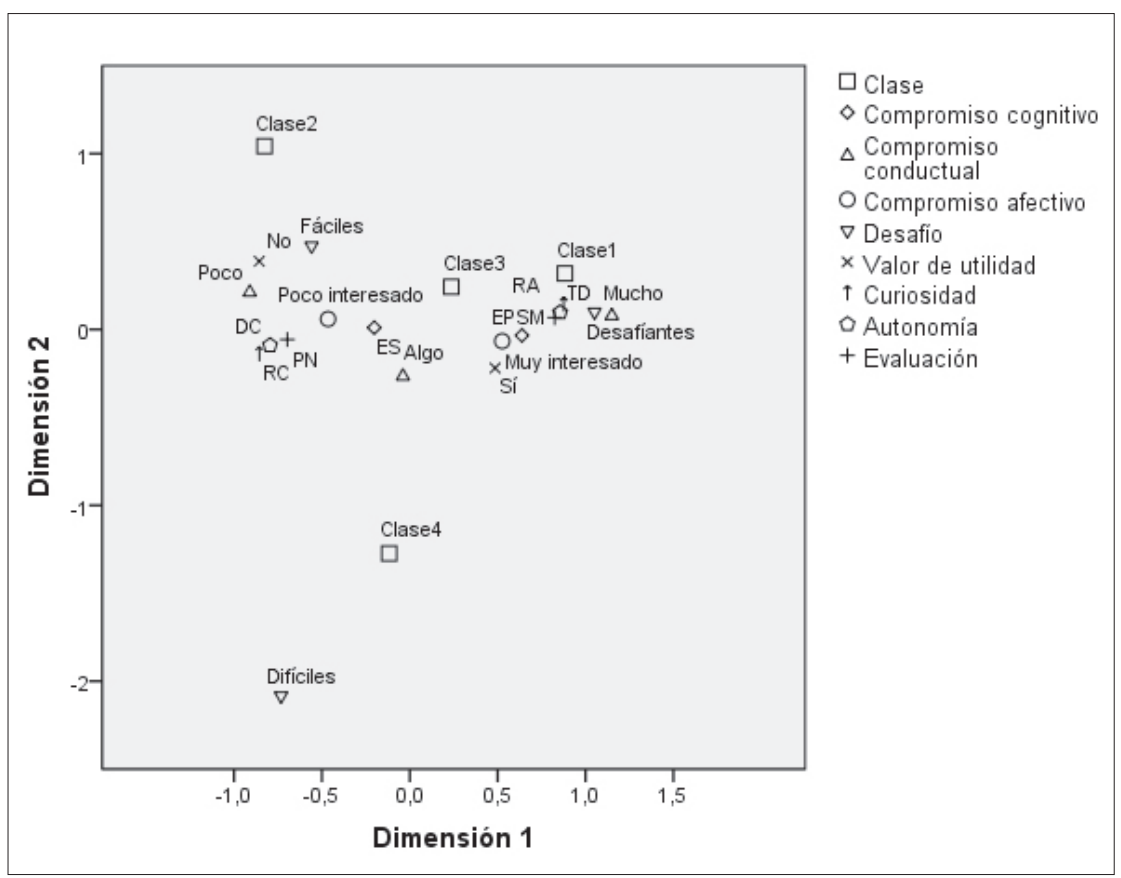




\section{CONSIDERACIONES FINALES}

El objetivo del presente trabajo fue explorar y describir, en una muestra de estudiantes de educación primaria, cómo las características de la clase promueven o no el compromiso cognitivo, afectivo y conductual, e influyen en la percepción que estos tienen del contexto instructivo. Según lo esperado, los resultados de este estudio indican que el compromiso de los estudiantes y la percepción que estos construyen en torno al contexto de enseñanza se relacionan significativamente con las características de la clase.

Más específicamente, hallamos que aquellos docentes que tienden a configurar la clase contemplando las seis dimensiones retroalimentación, soporte docente, oportunidad para aprender, participación, estructura de la clase, evaluación- que desde la teoría estarían facilitando la promoción del compromiso, son los entornos que se relacionan significativamente con un mayor compromiso cognitivo, afectivo y conductual de los alumnos que forman parte de ese contexto de enseñanza-aprendizaje. Asimismo, los alumnos que participan de clases con rasgos que posibilitan el desarrollo de la implicación, son aquellos que perciben al contexto académico como desafiante, con posibilidades de tomar decisiones, y buscan respuestas alternativas. Además, buscan otorgar sentido de utilidad al contenido enseñando y considerar la evaluación como una instancia para mejorar el proceso de aprendizaje. En este sentido, los datos sugieren que el compromiso se contagia en función de los rasgos que configuran la clase. Resultados que, en su análisis conjunto, muestran la complejidad y la riqueza de estudiarlas en interrelación para entender cómo explican el compromiso de los estudiantes en sus tres facetas.

Lo encontrado está en la línea de los postulados y estudios empíricos realizados por Harris (2010), Hipkins (2012), Lam et al. (2012), Opdenakker y Damme (2006), Reeve et al. (2004) y Schussler (2009), quienes, además, entienden que es necesario estudiar distintas dimensiones de la clase, el docente y los alumnos para comprender la complejidad que supone planificar prácticas tendientes a mejorar el compromiso hacia el aprendizaje. Asimismo, los estudios de Jang, Reeve y Deci (2010) y Guthrie, Wigfield y You (2012) muestran resultados acordes a las características de las clases observadas que interrelacionan con las percepciones del contexto instructivo que construyen los estudiantes más implicados cognitivamente, afectivamente y conductualmente.

Marcamos como fortaleza del estudio los avances realizados hacia un modelo de investigación integrada, que reclama volver al aula 
para interactuar con lo que ocurre ahí, orientados por teorías que guían la observación inicial y permiten una interpretación de los resultados que definirán líneas para una enseñanza más eficaz para alumnos y docentes. Y entendemos que futuros estudios deberán avanzar en las posibilidades de integrar los factores personales del alumno, que puedan estar relacionados con la promoción de una mayor implicación en sus tres dimensiones, tales como orientación motivacional, atribución de éxito y fracaso, entre otras, que permitan ampliar la comprensión y configuración de un entorno óptimo para el desarrollo del compromiso.

Asimismo, seráimportante contarconotras medidas alternativas al autorreporte de los estudiantes para evaluar el compromiso cognitivo y el afectivo, así como las variables del contexto instructivo, ya sea desarrollando indicadores de observación o bien promoviendo prácticas instruccionales dirigidas a potenciar el involucramiento que permitan confirmar más en profundidad los resultados hallados.

\section{REFERENCIAS}

ALEXANDER, P. Psychology in learning and instruction. New Jersey: Pearson Prentice Hall, 2006.

AMES, C. Classroom: Goals, structures, and student motivation. Journal of Educational Psychology, Northeast, v. 84, n. 3, p. 261-271, Jan. 1992.

APPLETON, J. et al. Measuring cognitive and psychological engagement: validation of the student engagement instrument. Journal of School Psychology, Dekald, v. 44, n. 5, p. 427445, May 2006.

ARGUEDAS, I. Involucramiento de los estudiantes y los estudiantes en el proceso educativo. Revista Electrónica Iberoamericana sobre Calidad, Eficacia y Cambio en Educación, Madrid, v. 8, n. 1, p. 63-78, oct. 2010.

BANDURA, A. Social foundations of thought and action: a social cognitive theory. Englewood Cliffs: Prentice-Hall, 1986.

BLUMENFELD, P.; KEMPLER, T.; KRAJCIK, J. Motivation and cognitive engagement in learning environments. In: SAWYER, R. K. (Ed.). The Cambridge Handbook of the learning science. New York: Cambridge University Press, 2006. p. 475-488.

CASTORINA, J. A. et al. Piaget en la educación: debate en torno a sus aportaciones. México: Paidós: Universidad Autónoma de México, 1998.

ECCLES, J. et al. Expectancies, values, and academic behaviors. In: SPENCE, J. (Ed.). Achievement and achievement motives. San Francisco: W. H. Freeman, 1983. p. 75-146. ECCLES, J.; WIGFIELD, A. Motivational beliefs, values, and goals. Annu. Rev. Psychol., Palo Alto, v. 53, n. 1, p. 109-132, Feb. 2002.

FINN, J. Introduction. In: J, FINN. School engagement $\&$ student at risk. Washington, DC: National Center for Educational Statistics, 1993. p. 01-21. Disponible en: < http:/ / nces. ed.gov/pubs93/93470a.pdf.>. Consultado el: 06 Sept. 2014. 
FINN, J.; ZIMMER, K. Student engagement: what is it? Why does it matter? In: CHRISTENSON, S., RESCHLY, A.; WYLIE C. (Ed.). Handbook research on student engagement. New York: Springer, 2012. p. 97-131.

FREDRICKS, J. A.; MCCOLSKEY, W. The measurement of student engagement: a comparative analysis of various methods and student self-report instruments. In: CHRISTENSON, S.; RESCHLY, A.; WYLIE, C. (Ed.). Handbook research on student engagement. New York: Springer, 2012. p. 763-782.

FREDRICKS, J. A.; BLUMENFELD, O.; PARIS, A. School engagement: potential of the concept state of the evidence. Review of Educational Research, Thousand Oaks, v. 74, n. 1, p. 59-109, Mar. 2004.

GONZÁLEZ GONZÁLEZ, M. T. El alumno ante la escuela y su propio aprendizaje: algunas líneas de investigación en torno al concepto de implicación. Revista Iberoamericana sobre Calidad, Eficacia y Cambio en Educación, Madrid, v. 8, n. 4, p. 10-31, jun. 2010.

GOOD, T. Forty years of research on teaching 1968-2008: what do we know now that we didn't know then?. In: MARZANO, R. J. (Ed.). On excellence in teaching. Bloomington: Solution Tree Press, 2010. p. 31-64.

GUTHRIE, J.; WIGFIELD, A.; YOU, W. Instructional contexts for engagement and achievement in reading. In: CHRISTENSON, S.; RESCHLY, A.; WYLIE, C. (Ed.). Handbook research on student engagement. New York: Springer, 2012. p. 601-634.

HARRIS, L. Delivering, modifying or collaborating? Examining three teacher conceptions of how to facilitate student engagement. Teachers and Teaching, Abingdon, v. 16, n. 1, p. 131-151, Nov. 2010.

HIPKINS, R. The engaging nature of teaching for competency development. In: CHRISTENSON, S.; RESCHLY, A.; WYLIE, C. (Ed.). Handbook research on student engagement. New York: Springer, 2012. p. 441-456.

HUGHES, J.; WU, W.; WEST, S. Teacher performance goal practices and elementary students' behavioral engagement: a developmental perspective. Journal of School Psychology, Columbus, v. 49, n. 1, p. 1-23, Feb. 2011.

JANG, H.; REEVE, J.; DECI, E. Engaging students in learning activities: it is not autonomy support or structure but autonomy support and structure. Journal of Educational Psychology, Northeast, v. 102, n. 3, p. 588-600, Aug. 2010.

KONG, Q.; WONG N.; LAM, C. Student engagement in mathematics: development of instrument and validation of construct. Mathematics Education Research Journal, Clayton, v. 15, n. 1, p. 4-21, May 2003.

LAM, S.; PAK, T.; MA, W. Motivating instructional contexts. In: ZELICK, P. (Ed.). Issues in the psychology of motivation. New York: Nova Science Publishers, 2007. p. 119-136.

LAM, S. et al. Understanding student engagement with a contextual model. In: CHRISTENSON, S.; RESCHLY, A.; WYLIE, C. (Ed.). Handbook research on student engagement. New York: Springer, 2012. p. 403-419.

MITCHELL, I.; CARBONE, A. A typology of task characteristics and their effects on student engagement. International Journal of Educational Research, Amsterdam, v. 50, n. 5-6, p. 257-270, June 2011.

NEWMANN, F.; WEHLAGE, G.; LAMBORN, S. The significance and sources of student engagement. In: NEWMANN D. M. (Ed.). Student engagement and achievement in american secondary schools. New York: Teachers College Press, 1992. p. 11-39. 
NICHOLS, S.; DAWSON, H. Assessment as a context for student engagement. In: CHRISTENSON, S.; RESCHLY, A.; WYLIE, C. (Ed.). Handbook research on student engagement. New York: Springer, 2012. p. 457-477.

OPDENAKKER, M.; DAMME, J. van. Teacher characteristics and teaching styles as effectiveness enhancing factors of classroom practice. Teaching and Teacher Education, Amsterdam, v. 22, n. 1, p. 1-21, Jan. 2006.

PIANTA, R.; HAMRE, B.; ALLEN, J. Teacher-Student relationships and engagement: conceptualizing, measuring, and improving the capacity of classroom interactions. In: CHRISTENSON, S.; RESCHLY, A.; WYLIE, C. (Ed.). Handbook research on student engagement. New York: Springer, 2012. p. 365-386.

REEVE, J. et al. Enhancing students' engagement by increasing teachers' autonomy Support. Motivation and Emotion, Cham, v. 28, n. 2, p. 147-169, Dec. 2004.

RIGO, D. Y. Compromiso hacia las tareas académicas: diseños instructivos e inteligencias múltiples. Ikastorratza, e-Revista Didáctica, Leioa, v. 10, n. 1, jul. 2013. Disponible en: <http://www.ehu.es/ikastorratza/10_alea/tareas.pdf>. Consultado el: 09 sep. 2014.

RIGO, D. Y. Enseñar y aprender: promoción del compromiso, diseño instructivo e inteligencias múltiples. In: ALONSO ROQUE, J. I.; MARTÍNEZ-ARTERO, R. N. (Ed.). Investigación educativa en educación primaria. Universidad de Murcia: Edit.um, 2014. p. 320-336.

RIGO, D. Y.; DONOLO, D. Entre pupitres y pizarrones. Retos en educación primaria: el aprendizaje con compromiso. Educatio Siglo XXI, Murcia, v. 32, n. 2, p. 59-80, mar. 2014.

RUHE, V. A toolkit for writing surveys to measure student engagement, reflective and responsible learning. Minneapolis: Centre for Teaching and Learning Services, University of Minnesota, 2007.

RYAN, A.; PATRICK, H. The classroom social environment and changes in adolescents' motivation and engagement during middle school. American Educational Research Journal, Thousand Oaks, v. 38, n. 2, p. 437-460, June 2001.

SCHUNK, D.; PAJARES, F. Self-efficacy in education revisited: empirical and applied evidence. In: MCINERNEY, D. M.; ETTEN, S. van. (Ed.). Sociocultural influences on motivation and learning. Greenwich: Information Age Publishing, 2004. p. 115-138.

SCHUSSLER, D. Beyond Content: How teachers manage classrooms to facilitate intellectual engagement for disengaged students. Theory Into Practice, Nueva York, v. 48, n. 2, p. 114121, Oct. 2009.

SHERNOFF, D. Optimal learning environments to promote student engagement. New York: Springer, 2013.

SMITH, M. Differentiated instruction and teacher flow. Minnesota: St. Mary's University of Minnesota, 2009 apud SHERNOFF, D. Optimal learning environments to promote student engagement. New York: Springer, 2013.

STIPEK, D. Motivation and instruction. In: BERLINER, D.; CALFEE, R. (Ed.). Handbook of educational psychology. New York: Simon \& Schuster Macmillan, 1996. p. 85-113.

TURNER, J. C.; MEYER, D. K. Studying and understanding the instructional contexts of classroom: using our past to forge our future. Educational Psychologist, New York, v. 35, n. 2, p. 69-85, June 2000. 


\section{NOTAS}

${ }^{1}$ Las variables representadas son: configuración de la clase (clases 1, 2, 3 y 4); compromiso cognitivo (ES o estrategias superficiales - EP o estrategias profundas); compromiso conductual (mucho - algo - poco); compromiso afectivo (muy interesado - poco interesado); desafío (desafiantes, fáciles - difíciles); valor de utilidad (sí - no); curiosidad (RC o respuesta correcta - RA o explorar y encontrar respuestas alternativas); autonomía (DC o delimitarte en lo que la consigna - TD o tomar decisiones); evaluación (PN o poner una nota - SM o sugerencias para mejorar el trabajo).

Submetido: 09/10/2015

Aprovado: 26/10/2016

Contato:

Daiana Yamila Rigo

Colón 351, San Basilio,

Córdoba, Argentina.

C.P. 5841 
